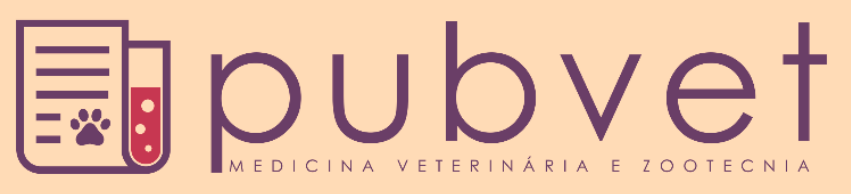

https://doi.org/10.31533/pubvet.v14n12a702.1-6

\title{
A avaliação bioquímica do sistema urinário como ferramenta diagnóstica: Revisão
}

\author{
Mylena Ellen de Sousa Freitas ${ }^{10}$, Edilene Pereira de Sousa ${ }^{1}{ }^{\ominus}$, Carolina Coutinho Beserra $^{1}{ }^{\theta}$, \\ Magda da Silva Cavalcante ${ }^{10}$, Beatriz Evaristo de Almeida Tabosa ${ }^{10}$, Breno Queiroz Pinheiro ${ }^{2 *}$ \\ ${ }^{1}$ Discente do curso de Medicina Veterinária, Universidade Estadual do Ceará, Faculdade de Veterinária, Ceará, Brasil \\ ${ }^{2}$ Docente da Universidade Estadual do Ceará, Faculdade de Veterinária, Ceará, Brasil \\ *Autor para correspondência,E-mail: breno.queiroz@uece.br
}

Resumo. A avaliação do perfil bioquímico renal sérico é uma metodologia rotineiramente utilizada, muitas vezes em conjunto com outros exames, como a urinálise e a ultrassonografia, para avaliar o sistema urinário. Por meio dela, é possível realizar o diagnóstico de doenças renais, bem como fazer o acompanhamento da progressão dos tratamentos instituídos. Desta forma, o objetivo do presente trabalho é fazer uma revisão de literatura acerca da bioquímica urinária e de sua utilização no diagnóstico de distúrbios renais, através da avaliação de diferentes marcadores renais. Realizou-se um estudo descritivo analítico, desenvolvido através de pesquisa do tipo bibliográfica, utilizando-se da análise de livros, revistas, publicações especializadas e artigos, datados a partir de janeiro de 2010. Para tanto, foram utilizadas ferramentas digitais, como a PubVet, o Google Acadêmico, Periódicos Capes, Scielo e Science direct. Concluiu-se que os biomarcadores creatinina e ureia, ainda são os mais utilizados na rotina clínica veterinária. O SDMA é um novo biomarcador mais precoce que creatinina e ureia, que vem demonstrando bons resultados nos diagnósticos de doença renal aguda e doença renal crônica.

Palavras chave: Biomarcadores renais, creatinina, dimetilarginina simétrica, ureia

\section{Biochemical assessment of the urinary system as a diagnostic tool: Review}

Abstract. The evaluation of the serum renal biochemical profile is a methodology routinely
used, often in conjunction with other tests, such as urinalysis and ultrasound, to assess the
urinary system. Through this is possible to make the diagnosis of kidney diseases, as well
as monitor the progression of the established treatments. The aim of the present study is to
review the literature on urinary biochemistry for the diagnosis of renal disorders, through
the assessment of different renal markers. In the present work, a descriptive analytical study
was carried out, developed through bibliographic research, using the analysis of books,
magazines, specialized publications and articles, dated since January 2010 . For this
purpose, digital tools were used, such as PubVet, Google Scholar, Capes Journals, Scielo
and Science direct. It was concluded that the creatinine and urea biomarkers are still the
most used in the veterinary clinical routine. SDMA is a new biomarker earlier than
creatinine and urea, which has been showing good results in the diagnosis of acute kidney
disease and chronic kidney disease.

Keywords: Renal biomarkers, creatinine, symmetrical dimethylarginine, urea 


\section{Evaluación bioquímica del sistema urinario como herramienta de diagnóstico: Revisión}

Resumen. La evaluación del perfil bioquímico renal sérico es una metodología utilizada
habitualmente, a menudo junto con otras pruebas, como análisis de orina y ultrasonido,
para evaluar el sistema urinario. A través de él, es posible hacer el diagnóstico de
enfermedades renales, así como monitorear la progresión de los tratamientos establecidos.
Por lo tanto, el objetivo del presente trabajo es revisar la literatura sobre bioquímica urinaria
y su uso en el diagnóstico de trastornos renales, a través de la evaluación de diferentes
marcadores renales. Se realizó un estudio analítico descriptivo, desarrollado a través de la
investigación bibliográfica, utilizando el análisis de libros, revistas, publicaciones
especializadas y artículos, con fecha de enero de 2010 . Para este propósito, se utilizaron
herramientas digitales, tales como PubVet, Google Scholar, Capes Journals, Scielo y
Science direct. Se concluyó que los biomarcadores de creatinina y urea siguen siendo los
más utilizados en la rutina clínica veterinaria. SDMA es un nuevo biomarcador precoz que
la creatinina y la urea, que ha mostrado buenos resultados en el diagnóstico de enfermedad
renal aguda y enfermedad renal crónica.

Palabras clave: Biomarcadores renales, creatinina, dimetilaginina simétrica, urea

\section{Introdução}

A avaliação do perfil bioquímico sérico é uma metodologia rotineiramente utilizada, muitas vezes em conjunto com outros exames, como a urinálise e a ultrassonografia, para avaliar o sistema urinário. Por meio dela, é possível realizar o diagnóstico de doenças renais, bem como fazer o acompanhamento da progressão dos tratamentos instituídos (

A taxa de filtração glomerular (TFG) é o parâmetro mais confiável para avaliar a função renal, mas, na medicina veterinária, não é comum que se faça essa mensuração. Todavia, a TFG pode ser indiretamente avaliada por meio da análise sérica de certos compostos, que permitem fazer uma estimativa da diminuição da TFG. Nesse contexto, dentre os biomarcadores mais usuais, a creatinina é considerada um melhor indicador para estimar a TFG porque, ao contrário da ureia, não sofre influência significativa de fatores extra renais (Thrall, 2015).

A creatinina e a ureia aumentam conforme a TFG diminui, porém, esse aumento só ocorre quando a TFG já foi diminuída em pelo menos $75 \%$ e, devido a isso, esses compostos não podem ser considerados como marcadores precoces de doença renal (Thrall, 2015). O aumento da concentração desses compostos nitrogenados não proteicos é denominado azotemia e esta pode ser classificada em pré-renal, renal e pós-renal, a depender da sua origem (Nelson \& Couto, 2015).

Devido à grande relevância das doenças renais, nos últimos anos intensificou-se a busca por marcadores renais que detectem de forma mais precoce alterações na função renal. A dimetilarginina simétrica (SDMA) tem se mostrado como um eficiente biomarcador, devido a sua alta sensibilidade, o que permite detectar alterações no funcionamento dos rins mais precocemente que os analitos anteriormente citados (Hall et al., 2014a).

O presente trabalho tem como objetivo fazer uma revisão de literatura acerca da bioquímica urinária e de sua utilização no diagnóstico de distúrbios renais, através da avaliação dos principais marcadores renais.

\section{Material e métodos}

No presente trabalho foi realizado levantamento bibliográfico em livros e artigos especializados, datados a partir de janeiro de 2010, elaborando uma revisão de literatura, na qual expõe-se a importância dos achados relacionados à bioquímica urinária como ferramentas diagnósticas. As ferramentas digitais utilizadas foram plataformas de busca online, como o Google Acadêmico, Periódicos Capes, Scielo e Science Direct. 


\section{Creatinina}

A creatinina é o produto residual da creatina e do fosfato de creatina, ambos encontrados no músculo. Sua produção é constante e proporcional à massa muscular do animal (Cannon, 2016). A creatinina sérica é o marcador endógeno mais amplamente utilizado para estimar a TFG, ela é melhor indicador da TFG do que a ureia, pois a velocidade de produção da creatinina e sua excreção são relativamente constantes, além de não ser metabolizada por processos extrarenais (Thrall, 2015). Redução da TFG causa retenção de creatinina e, portanto, acarreta na elevação do seu valor sérico (Relford et al., 2016).

É amplamente aceito que pelo menos $75 \%$ da massa de néfrons deve ser perdida para que os valores de referência de creatinina estejam aumentados (Hokamp \& Nabity, 2016), podendo, dessa forma, ser utilizada tanto para a doença renal crônica quanto para a lesão renal aguda (Macedo, 2011). No entanto, deve ser analisado com cautela, devido ao fato de que sua concentração poder sofrer variações por fatores não renais como sexo, idade, peso corporal, índice de hidratação, massa e metabolismo muscular, estado nutricional e anorexia (Vaidya et al., 2008).

Dietas proteicas (ricas em creatina) podem aumentar a creatinina sérica, devido ao aumento da absorção gastrintestinal, isso é compensado pelo aumento da TFG estimulado pela refeição. O aumento de creatinina de origem alimentar (carne) é insignificante, pois o aumento pós-prandial da TFG, na verdade, faz com que ocorra diminuição na concentração sérica de creatinina 2 horas após a refeição (Thrall, 2015). É importante lembrar que a desidratação e as alterações na massa muscular possuem efeito sobre o valor da creatinina sérica, fazendo com que o estado de hidratação e a massa muscular devam ser considerados na interpretação desse parâmetro (Relford et al., 2016).

$\mathrm{O}$ valor da creatinina tem relação direta com o metabolismo muscular, a função renal pode ser subestimada em animais com baixo escore corporal ou superestimada em animais muito musculosos (Yerramilli et al., 2016). Este fato foi demostrado por Hall et al. (2014b), onde em um estudo com trinta e dois gatos saudáveis, com idade média de 14,0 $(8,3$ a 19,6) o valor da creatinina e a TFG foram menores em gatos com mais de 15 anos de idade em comparação com gatos com menos de 15 anos. Geralmente em animais idosos a massa muscular pode ser significativamente reduzida, assim os valores de creatinina desses pacientes podem ser menores que os valores de referência da população (Giraldi et al., 2020).

Uma brusca diminuição na TFG produz apenas um pequeno aumento nos valores de creatinina sérica de pacientes com doença inicial, enquanto, em pacientes mais crônicos, pequenas alterações na TFG resultam em grandes elevações na creatinina (Braff et al., 2014). Logo, pequenos aumentos na creatinina sérica podem indicar um dano significativo da função renal de pacientes aparentemente saudáveis. E uma gradual elevação na creatinina, mesmo que ainda dentro dos valores de referência, com diminuições na densidade urinária (DU) abaixo de 1.030, pode ser indicativo de insuficiência renal (Cannon, 2016).

\section{Ureia}

A ureia é o segundo biomarcador renal mais utilizado na prática clínica e, assim como a creatinina, também só avalia, tardiamente, as lesões renais (Nascimento et al., 2017). No entanto, esse composto não é específico e pode sofrer variações em seus valores em decorrência de fatores extra renais (Dalton, 2011). Este é um metabólito tóxico nitrogenado resultante da degradação de proteínas que se inicia na proteólise, havendo sua biossíntese nos hepatócitos, a partir da amônia que é um composto nitrogenado mais tóxico, obtida em decorrência da degradação proteica, havendo posteriormente sua excreção pelo sistema urinário. Apesar de ser filtrado pelos glomérulos esse composto sofre um processo de difusão passiva dependendo do fluxo urinário, no qual esse componente passa de um local de maior concentração para um de menor concentração, podendo ser reabsorvido pós a filtração em cerca de $20 \%$ (Hokamp \& Nabity, 2016).

O transporte de ureia pelo túbulo proximal não é regulado pela vasopressina (também chamado de hormônio antidiurético), mas é aumentada com o aumento do transporte de sódio. O túbulo contorcido distal tem uma baixa permeabilidade à ureia. No entanto, alguma ureia é reabsorvida nesse segmento. Os ductos coletores medulares corticais e externos têm baixa permeabilidade à ureia. Por outro lado, a medula interna do ducto coletor (MIDC) tem um alta permeabilidade à ureia, que nesse caso é aumentada pela vasopressina. Existe extensa reabsorção de ureia pela MIDC no lúmen do interstício (Weiner et al., 2015). 
Logo, vale ressaltar sua inespecificidade, pois sua concentração pode aumentar em casos de desidratação, onde ocorre maior reabsorção da ureia, e também em animais com hemorragia gastrointestinal, onde a fração proteica da hemácia é convertida em amônia pelas bactérias locais, sendo reabsorvida pelo fígado e transformada em ureia, podendo também diminuir em algumas condições, como na insuficiência hepática e na desnutrição proteica (Nascimento et al., 2017). Dessa forma, para minimizar alterações na sua dosagem, é importante que tanto a creatinina quanto a ureia sejam dosadas simultaneamente, em jejum, para avaliar a existência de danos renais e devem ser avaliadas de forma seriada diante da suspeita de lesão aguda ou crônica (Nascimento et al., 2017).

Em ruminantes, devido a sua flora ruminal, que degrada a ureia em aminoácidos e assimila-os, resultando em ganho proteico, torna-se especialmente complicado correlacionar a ureia nitrogenada à creatinina e à densidade específica urinária para predizer doenças renais nessa espécie. Inclusive a correlação do aumento sérico da ureia sem aumento concomitante da creatinina tem sido utilizada para a identificação de possível estase ruminal e sangramento gastrointestinal. Nos equinos, apesar excreção de ureia pelo sistema gastrointestinal, a sua concentração pode ser utilizada para avaliação da função renal (Thrall, 2015).

Fitas ou tiras reagentes que utilizam urease para estimar a concentração de nitrogênio ureico são menos precisas do que as fitas que utilizam o método da amônia, que é um método de determinação semiquantitativo. Esses métodos são úteis para dar uma ideia global da estimativa de "normalidade" ou do "aumento" da ureia sérica quando outros exames não estiverem disponíveis. Deve-se saber que esses exames por fitas reagentes não são precisos e que não devem ser utilizados para monitorar pacientes por longos períodos, sendo úteis apenas para identificar concentrações de baixas a normais, pois não são capazes de quantificar valores superiores à normalidade. Caso a tira indique azotemia, então amostras sanguíneas devem ser coletadas antes da fluidoterapia e o soro deve ser avaliado por metodologia bioquímica quantitativa (Thrall, 2015).

Vale ressaltar que alterações nos níveis séricos de creatinina e ureia também podem indicar além das doenças renais, outras diferentes etiologias clínicas, sendo de maneira primária na leptospirose ou secundária na gastroenterite, na intoxicação vegetal animal e nas mortes em animais portadores de leishmaniose, além dos fatores já conhecidos pré e pós-renais. Por isso, a avaliação laboratorial da creatinina e ureia se faz necessária também na prevenção e manutenção da saúde dos animais. Apesar de estudos recentes indicarem investimentos na descoberta precoce de novos marcadores de disfunções, os níveis de creatinina e ureia utilizados na rotina clínica laboratorial são efetivos como marcadores indiretos da TFG em animais (

\section{Dimetilarginina simétrica (SDMA)}

Dimetilarginina Simétrica (SDMA) é um biomarcador renal descoberto relativamente recente. SDMA é primariamente eliminado pela excreção renal, não sofrendo influência de mecanismos extra renais. Portanto, é um marcador endógeno da TFG. Não é influenciado pela massa muscular, o que é uma vantagem em comparação com a creatinina. É um importante biomarcador para detecção precoce de disfunção renal, em estudos de nefrologia humana, em pacientes com Lesão Renal Aguda (LRA). No entanto, não existem informações suficientes do uso de SDMA em casos de lesão renal aguda (LRA) (Dahlem et al., 2017).

Sendo o SDMA uma molécula originada da metilação intranuclear do aminoácido arginina, sendo liberada na circulação após a proteólise. Seu peso molecular é de $202 \mathrm{~g} / \mathrm{mol}$ e sua carga é positiva permitindo que seja livremente filtrada pelo glomérulo (Relford et al., 2016). Mais de $90 \%$ da sua eliminação ocorre por excreção renal, o que faz que a mensuração de seu valor sérico possa ser um marcador endógeno da TFG Hall et al. (2014a).

Foi realizado um estudo, por Hall et al. (2014a), para mostrar a utilidade de medir as concentrações séricas de SDMA para detectar Doença Renal crónica (DRC) em gatos antes do diagnóstico por medições convencionais de aumento da creatinina sérica. Com isso foram utilizadas amostras de gatos antes de se tornarem azotêmicos, contanto que fosse possível utilização do soro para medições de SDMA e creatinina sérica. Sendo utilizados gatos de diversas idades, com alimentações diferentes, ambientes diferentes e hábitos diferentes. Sendo utilizados 21 gatos com DRC e 21 gatos saudáveis geriátricos. 
Ainda neste estudo, usando amostras retrospectivas de soro, puderam mostrar que o SDMA sérico aumenta antes da creatinina sérica em gatos com DRC. As amostras de soro foram depositadas como parte de exames anuais ou como parte de protocolos para outros estudos. Portanto, os dados de intervalo exato não estavam disponíveis. No entanto, as concentrações séricas de SDMA aumentaram acima do intervalo de referência normal antes que as concentrações de creatinina sérica aumentassem acima do intervalo de referência normal em uma média de 14,6 meses (variação de 0 a 48 meses) em 21 gatos diagnosticados com DRC (Hall et al., 2014a). A mensuração do SDMA em felinos assintomáticos mostrou-se capaz de estabelecer o diagnóstico precoce da doença renal e com isso se instituir cuidados que virão a reduzir a evolução da doença. Contudo, estudos futuros são necessários para fortificar tais resultados (Hall et al., 2014a).

Posteriormente, Dahlem et al. (2017) fizeram estudo com cães, cujo objetivos foram relatar a concentração de SDMA em cães com LRA ou Doença Renal Aguda (DRA) - e investigar se SDMA pode ser um biomarcador adequado para diferenciar DRA da DRC. Em segundo lugar, tiveram como objetivo avaliar se SDMA pode ter valor prognóstico em cães com LRA. Para isso, foram mensuradas concentrações de SDMA plasmático em um total de 95 cães. Incluindo 18 cães saudáveis, 48 cachorros com Lesão Renal Aguda e 28 cachorros com Doença Renal Crônica. Observou-se que as concentrações de creatinina e SDMA foram significativamente maiores em cães com azotemia renal em comparação com cães saudáveis. Tanto a creatinina quanto o SDMA são marcadores da TFG e se correlacionam inversamente com a concentração de taxa de filtração glomerular. Cães com LRA têm concentração de SDMA plasmática semelhante à de cães com DRC, e acentuada elevação da concentração de SDMA comparada a cães saudáveis. Nesta população de cães utilizada no estudo, a relação SDMA/creatinina foi significativamente menor no grupo com LRA em comparação com o grupo com DRC por razões ainda desconhecidas.

O teste SDMA parece realmente facilitar o diagnóstico precoce da DRC, no entanto os veterinários ainda não possuem muita familiaridade com o exame, e o mesmo não está acessível a todas as clínicas veterinárias no Brasil. Somente quando seu uso for mais difundido, houver mais estudos e uma maior experiência em sua utilização ele poderá passar mais segurança aos clínicos que o utilizarem (Vieira, 2018).

\section{Considerações finais}

Com o presente estudo, percebe-se que os biomarcadores creatinina e ureia, mesmo que tardios, ainda são os mais utilizados na rotina clínica veterinária, e podem ser utilizados tanto para detecção de doenças renais como para outras patologias. Já o SDMA é um novo biomarcador mais precoce que creatinina e ureia, que vem demonstrando bons resultados nos diagnósticos de Doença Renal Aguda e Doença Renal Cránica, porém, ainda pouco utilizado na rotina veterinária e que precisa de mais estudos para conhecer o seu potencial diagnóstico.

\section{Referências bibliográficas}

Braff, J., Obare, E., Yerramilli, M., Elliott, J., \& Yerramilli, M. (2014). Relationship between serum symmetric dimethylarginine concentration and glomerular filtration rate in cats. Journal of Veterinary Internal Medicine, 28(6), 1699-1701. DOI: https://doi.org/10.1111/jvim.12446

Cannon, M. (2016). Diagnosis and investigation of chronic kidney disease in cats. In Practice, 38(Suppl 3), 2-9. DOI: https://doi.org/10.1136/inp.i4914

Dahlem, D. P., Neiger, R., Schweighauser, A., Francey, T., Yerramilli, M., Obare, E., \& Steinbach, S. M. L. (2017). Plasma symmetric dimethylarginine concentration in dogs with acute kidney injury and chronic kidney disease. Journal of Veterinary Internal Medicine, 31(3), 799-804. DOI: https://doi.org/10.1111/jvim.14694

Dalton, R. N. (2011). Creatinina sérica e taxa de filtração glomerular: percepção e realidade. Jornal Brasileiro de Patologia e Medicina Laboratorial, 47(1), 8-11. DOI: https://doi.org/10.1590/s1676$\underline{24442011000100001}$

Giraldi, M., Paltrinieri, S., \& Scarpa, P. (2020). Electrophoretic patterns of proteinuria in feline spontaneous chronic kidney disease. Journal of Feline Medicine and Surgery, 22(2), 114-121. DOI: https://doi.org/10.1177/1098612X19827597 
Hall, J. A., Yerramilli, M., Obare, E., Yerramilli, M., \& Jewell, D. E. (2014a). Comparison of serum concentrations of symmetric dimethylarginine and creatinine as kidney function biomarkers in cats with chronic kidney disease. Journal of Veterinary Internal Medicine, 28(6), 1676-1683. DOI: https://doi.org/10.1111/jvim.12445

Hall, J. A., Yerramilli, M., Obare, E., Yu, S., \& Jewell, D. E. (2014b). Comparison of serum concentrations of symmetric dimethylarginine and creatinine as kidney function biomarkers in healthy geriatric cats fed reduced protein foods enriched with fish oil, L-carnitine, and medium-chain triglycerides. The Veterinary Journal, 202(3), 588-596. DOI: https://doi.org/10.1016/j.tvjl.2014.10.021

Hokamp, J. A., \& Nabity, M. B. (2016). Renal biomarkers in domestic species. Veterinary Clinical Pathology, 45(1), 28-56. DOI: https://doi.org/10.1111/vcp.12333

Macedo, E. (2011). Biomarcadores na insuficiência renal aguda. In H. Abensur (Ed.), Biomarcadores na Nefrologia. Roche.

Nascimento, M. R., Lima, C. S., cristina de Barros, J., Padovan, M., Cintra, C. A., Ayer, I. M., Borges, L. P. B., Pereira, A. G., \& Crivellenti, L. Z. (2017). Conceitos e aplicabilidade dos principais biomarcadores na nefrologia veterinária - revisão de literatura. Investigação, 16(8), 37-43. DOI: https://doi.org/10.26843/investigacao.v16i8.1863

Nelson, R. W., \& Couto, C. G. (2015). Medicina interna de pequenos animais (Issue 1). Elsevier Editora.

Ramos, G., \& Marini, D. C. (2015). Exames bioquímicos relacionados a alterações renais. Foco: Caderno de Estudos e Pesquisas, 6, 1-26.

Relford, R., Robertson, J., \& Clements, C. (2016). Symmetric dimethylarginine: improving the diagnosis and staging of chronic kidney disease in small animals. Veterinary Clinics: Small Animal Practice, 46(6), 941-960. DOI: https://doi.org/10.1016/j.cvsm.2016.06.010

Thrall, M. A. (2015). Hematologia e Bioquímica Clínica Veterinária. In 2. ed. Editora Roca.

Vaidya, V. S., Ferguson, M. A., \& Bonventre, J. V. (2008). Biomarkers of acute kidney injury. Annual Review Pharmacology Toxicology, 48, 463-493. DOI: https://doi.org/10.1016/B978-0-12-8012383.64110-7

Vieira, A. N. L. S. (2018). Concentração sérica de dimetilarginina simétrica (sdma) em cães com doença renal crônica submetidos a hemodiálise intermitente. 2018. 77 f. Dissertação (Mestrado) - Curso de Medicina Veterinária, Universidade Estadual Paulista Faculdade de Medicina Veterinária e Zootecnia, Botucatu.

Weiner, I. D., Mitch, W. E., \& Sands, J. M. (2015). Urea and ammonia metabolism and the control of renal nitrogen excretion. Clinical Journal of the American Society of Nephrology, 10(8), 1444-1458. DOI: https://doi.org/10.2215/CJN.10311013

Yerramilli, M., Farace, G., Quinn, J., \& Yerramilli, M. (2016). Kidney disease and the nexus of chronic kidney disease and acute kidney injury: the role of novel biomarkers as early and accurate diagnostics. Veterinary Clinics: Small Animal Practice, 46(6), 961-993. DOI: https://doi.org/10.1016/j.cvsm.2016.06.011.

\section{Histórico do artigo:}

Recebido: 24 de junho, 2020.

Aprovado: 8 setembro, 2020 .

Disponível online: 28 de outubro, 2020.
Licenciamento: Este artigo é publicado na modalidade Acesso Aberto sob a licença Creative Commons Atribuição 4.0 (CC-BY 4.0), a qual permite uso irrestrito, distribuição, reprodução em qualquer meio, desde que o autor e a fonte sejam devidamente creditados. 